\title{
Sirenen \\ Lösungen des Klangs vom Körper
}

\author{
Philipp von Hilgers \\ Hermann v. Helmholtz-Zentrum für Kulturtechnik, Berlin
}

Was wir aber erreichen können, ist die Kenntnis der gesetzlichen Ordnung im Reiche des Wirklichen, diese freilich nur dargestellt in dem Zeichensystem unserer Sinneseindrücke.

Hermann von Helmholtz

Ganze Kriegsgenerationen, zumal in Deutschland, kennen Sirenen fast nur von der Seite ihrer durchdringenden Wirkung - ihre Wirkungsweise, ihre „Erfinder“, die Gründe, die zu ihrer Entwicklung geführt haben, dagegen kaum. "A screaming comes across the sky" heißt es im ersten Satz von Thomas Pynchons Weltkriegsepos „Gravity's Rainbow". Von autonom rechnenden Trägerraketen, die dem Schall vorauseilen und nahezu spurloser Destruktion preisgegeben sind, hat sich der Klang gelöst und eilt nur mit Schallgeschwindigkeit dem erloschenen Objekt seiner Verursachung hinterher. Der Umschlag vom Mythos zur Techné scheint gebahnt: In hochgerüsteten Zeiten erster elektrischer Analogrechner und Ultrakurzwellenempfänger bedürfen Klänge notwendiger Weise weder der Nähe noch der Sichtbarkeit schwingender Körper. Doch der Zweite Weltkrieg endet nicht im Geheul von Albert Speers Raketenprogramm. Der Krieg geht mit den genau den gleichen Klängen zu Ende, mit dem er auch anhebt: Im anfänglichen Blitzkrieg der deutschen Wehrmacht stürzen denn Sirenen in ihrer vorerst letzten Bestimmung vom Himmel. Es ist Ernst Udet - ein Kampfflieger des Ersten 
Weltkriegs, Kunstflieger der Filme der Weimarer Zeit und schließlich Generalflugzeugmeister im Zweiten Weltkrieg -, der Sirenen am Sturzkampfflugzeug vom Typ Ju 87, besser bekannt als Stuka, anbringen läßt [Deighton 1979, 208-209]; wohl auf Weisung des Oberbefehlshabers der Wehrmacht Adolf Hitler [Virilio 1984, 105-106].

Im Verbund mit der Stuka wird der Wirkungszusammenhang der Sirene so offensichtlich, daß man von einer unauföslichen Einheit sprechen kann. Die Stukas sorgen im Sturzflug für einen stetig anschwellenden Luftstrom, den die Sirene in Form von Luftstößen abgibt. Dabei bewirkt der Luftstrom den Antrieb einer Scheibe, die ihn wiederum durch eine Reihe von Öffnungen gleichen Abstands kurzzeitig und stoßartig entweichen läßst. Eine Serie von zunehmenden Schalldruckdifferenzen wird somit in immer kürzerer zeitlicher Abfolge moduliert. Der Ton steigt nicht nur immer höher an, sondern wird auch immer lauter, da - physikalisch gesehen - Frequenz und Amplitude mit der Fallgeschwindigkeit der Stukas zunehmen. Vom Boden aus betrachtet, oder besser gesagt, vernommen, tritt aber noch ein ganz anderes Phänomen hervor: Durch den sogenannten Dopplereffekt schraubt sich die Tonhöhe des Sirenenklangs noch zusätzlich höher. ${ }^{1}$ Ernst Mach hatte schon bei Zeiten diese Möglichkeit bedacht: Einen von der Höhe herabfallenden Chor, der auf E-Dur gestimmt wäre, würde man am Boden somit in F-Dur hören [Mach 1862, 334]. Sirenen im Verbund mit Stukas sind solche durch Sturzflüge transponierte Chöre. Die Wahrnehmung ihres Glissando extensiviert punktuelle Angriffe der Sturzkampfflieger über alle physischen Grenzen hinaus. Am Ende des Krieges sind "Teppichbombardierungen" in ihrer Ausbreitung genauso flächendeckend wie der Sirenenalarm, der sie ankündigt. Die Botschaft der Odyssee scheint folglich immer noch zu gelten: Die Warnung vor Sirenen und die Warnung durch Sirenen ist ein und derselbe unlösliche Bann.

Sirenen bewegen sich im Verbund mit Stukas durch die Luft, werden von ihr angetrieben, um nichts anderes als Luft zu modulieren. Ihr Reservoir ist endlos, ihr Klangkörper der grenzenlose Raum. Raumgreifender und totalitärer können Instrumentierungen kaum sein. Mechanische Sirenen sind aber keineswegs erst aus den Weltkriegen hervorgegangen, sondern aus Diskursen und Experimenten des 19. Jahrhunderts. Hundert Jahre zuvor erfolgt der Einbruch dreifacher Relativität: Bewegte Beobachter, bewegte Klangquellen und bewegte Trägermedien heben Phäno-

\footnotetext{
${ }^{1}$ Gehen nämlich von einer Klangquelle nicht nur Impulse oder Schallwellen aus, sondern wird sie selbst in die gleiche Richtung bewegt, dann treffen die Schallwellenfronten in kürzeren Abständen auf Ohren am Zielort und bewirken einen Frequenzanstieg.
} 
mene auf, ändern sie oder belassen sie in Ambivalenz, die Forscher um 1850 nicht zur Ruhe kommen lassen [Mach 1861, 123]. ${ }^{2}$

\section{Sirenenformationen}

Sirenen eröffnen Klänge, die nicht an die Erscheinung von Klangkörpern gebunden sind und statt dessen von Zeichensystemen ausgehen: Das gilt für den Gesang unsichtbarer Nymphen in Homers Odyssee genauso wie für die Klanggenerierung eines durch und durch szientifischen Instruments. Es sind immerhin noch die gleichen basalen Praktiken des Schreibens und Bezifferns, die so verschiedene Klangquellen mit demselben Wort namhaft machen. So lassen sich Sirenen, die mythischen wie die technischen, im selben Raum der Schrift und der Einschreibungen beleuchten. Wenn deshalb Hermann von Helmholtz in seiner Lehre von den Tonempfindungen als verbindliches klangliches Referenzsystem die Sirene an den Anfang stellt, dann gilt es zu bemessen, in wieweit eine Schrift längst zu dem Zeichensystem — das, wie noch zu zeigen ist, die Sirene ist - Fragen stellt und Antworten gibt. Einfach ein Klangkörper ist die Sirene keinesfalls. Vielmehr ist zu vermuten, daß Sirenen, auch in der streng technischen Form, nach Homer nun ein weiteres, radikales Mal durch ein Zeichensystem zu ihrer Form gefunden und wechselseitig ein Zeichensystem formiert haben.

Historische Tiefenschichten, die ein unlängst angelaufenes Forschungsprogramm freizulegen unternimmt, ${ }^{3}$ liefern hierzu die kontrastive Folie. $\mathrm{Zu}$ ihr zählen die zeichentheoretisch tiefliegenden Befunde, daß die Odyssee Homers - anders noch als die frühere Ilias - mit dem griechischen Vokalalphabet ihr Spiel treibt, von und mit Stimmen spricht oder singt, die losgelöst von der körperlichen Präsenz heroischer Gestalten auf eine Hörerschaft ihre spurenreiche Wirkung ausüben. Ohne die Kontinuität einer technischen Entwicklung zu behaupten, die von Homer bis Helmholtz führe, bleibt zu fragen, ob Sirenen von 800 v. Chr. und 1800 n.

\footnotetext{
${ }^{2}$ Wenn in Albert Einsteins spezieller Relativitätstheorie - zumindest in seiner populären Fassung - Eisenbahnen zur Ausstattung von Gedankenexperimenten gehören, dann waren um 1850 ganze Eisenbahnzüge noch realer Bestandteil von Experimentalsystemen zur Untersuchung des akustischen Doppelereffekts [Buijs-Ballot 1845]; auch wenn „Eisenbahnen“, wie Ernst Mach lakonisch anmerkt, „als Experimentirmittel nicht Jedermann zu Gebote" standen [Mach 1860, 549].

${ }^{3}$ Das von Friedrich Kittler geleitete Forschungsprogramm untersucht am Hermann von Helmholtz-Zentrum für Kulturtechnik innerhalb eines Forschungsbereichs, der der Verschränkung von Bild, Schrift und Zahl nachgeht, den Einsatz der Mathematik am Leitfaden des griechischen Vokalalphabets und der pythagoreischen Fundierung der Musik.
} 
Chr. mehr verbindet als ein recht freier Gebrauch einer Metapher. Denn wie schon die vokalalphabetische Schrift ermöglicht auch die technische Sirene, einen radikalen Sprung zwischen den sinnlichen Modalitäten des Auditiven und Visuellen zu unternehmen. Die Einheit eines Eindrucks, der von der technischen Sirene ausgeht, ist im vorhinein in zeichenhaft berechenbare und mechanisch regelbare Elemente zerlegt. Bemerkenswert ist zudem die Verschränkung musikalischer und mathematischer Zeichenkonzepte, die seit den Tagen der Pythagoreer alle Vorzeichenwechsel übersteht.

Vor diesem Hintergrund erscheint viel weniger erklärungsbedürftig, wie die Sirene Helmholtz schließlich und offensichtlich als ein Bezugssystem zur Umfassung klanglicher Dimensionen dienen kann. Vielmehr könnte mit Blick auf Helmholtz' Grundlagenforschung die Vorgeschichte nicht lang genug ausfallen, die aufzeigt, wie grundlegend der Wandel ist, der mit klanggenerierenden Systemen wie der Sirene zwar epistemologische Fragestellungen eröffnet, aber selbst nicht mehr in Frage gestellt wird. Daß der Maßstab, den Helmholtz mit der Sirene ausgibt, noch zuvor im Zentrum eines unauföslichen Disputs stand, wurde in der Forschung zwar registriert. Doch viel zu kurz greifen Kommentare, die nur Differenzen unter Forschern ausmachen, was die adäquaten Beschreibung und Berechnung akustische Phänomene angeht. Viel grundstürzender sind weder überhaupt die phänomenalen Bereiche und Ebenen klar zu fassen, noch der Einfluß und die Einflußgrößen, die die Medialität der Experimental- und Aufzeichnungssysteme ausüben. So wird schließlich die These zu erhärten sein, daß Helmholtz gar nicht einen wissenschaftlichen Streit klären kann, der vermeintlich um die Sache geht, sich aber als schiere Differenz von Dingen, Zeichen und Materialitäten entpuppt.

Statt dessen ist zu analysieren, wie Helmholtz die Lehre von den Tonempfindungen und mithin die Sirene in ein fachwissenschaftlich übergreifendes Forschungsfeld integriert. Wissenschaftshistorisch fällt damit nicht nur ein weiteres Mal das Licht auf einen Forscher, dessen universelles Wirken schon bei Zeiten gerühmt wird. Denn im Schatten dieses Lichts harrt bis heute der Verdacht, jede Wissenschaft reicht nur soweit, wie die Medien, die sie beherrscht oder von denen sie beherrscht wird. Egal ob Sinnesorgan, Experimentalsystem oder Untersuchungsgegenstand, egal ob Physik, Physiologie oder Psychologie: Von einer bestimmten Zeit an versucht Helmholtz in den jeweiligen Bereich ohne Unterschied mit Zeichensystemen Zeichensysteme freizulegen.

Sind Binnendifferenzierung und rekurrente Bezugnahmen homogener Zeichensysteme auf dem gleichen Niveau erst einmal eingekommen, dann gerät Überkommendes und Zukünftiges, also im Falle der Sirenen, 
was sie mal historisch waren (Homers Musen) und wozu noch zukünftig gut sein werden (Terror und Alarm), in größte, mit Worten kaum zu überspielende Spannungen. Man kann diese Seiten in der Geschichte der technischen Sirenen als ihre völlig anderen Seiten ausweisen - nur ob sie deshalb als etwas völlig anderes und unverbundenes abgetan werden können, bliebt fraglich. Bei allen Absicherungen und Referenzen, mit denen Aufzeichnungen Zeichensystemen beikommen können und technische Details und Finessen herbeiführen: Maßgebliche Anstöße bei der Erforschungen solcher Experimentalsysteme sind äußerliche und scheinen als erste und letzte Instanzen auf.

Schon die Initiation und Namensgebung der Sirene durch den an der École polytechnique geschulten Physiker Cagniard de la Tour beruhte auf einer technisch und experimentell streng umrissenen Hörsituation, in die der uneinholbare Klang einer menschliche Stimme einbricht und die gleichzeitig sicherstellt, daß alle bis dahin vertrauten Verweise auf menschliche Körper systematisch ausschlossen sind. Cagniard spielt mit seiner Namensgebung folglich nicht bloß nur auf einen Mythos an, sondern ruft im Namen der Sirene ein Klang- und Zeichensystem auf, das Stimmen in Absenz ihrer Körper festschreibt. Wie anders die Sirene Klang und Stimme in Beziehung zum Körper und zum Zeichen setzt, werden im folgenden nur die Kontrastierungen zu den ihr im 18. Jahrhundert vorangegangenen Sprechmaschinen und eine knappe historische Rekapitulation der Klangaufzeichnung erweisen können. Erst in der Sirene, so die These, finden Klangsynthese und Klanganalyse zu einer gemeinsamen Kodierungsform.

Aber auch auf den weiteren Stationen in der Entwicklung der Sirene bleibt die Insistenz des Stimmlichen in der Kontingenz der Klangmuster virulent. Georg Simon Ohm werden sie im Zuge der Berechnung als auditive Täuschungen vernehmlich und intelligibel. Vor diesem Hintergrund dringt Helmholtz nicht mehr nur auf tonale, sondern auch auf klangliche Unterscheidungen, d.h. auf Feststellungen von „Klangfarben“, und analysiert experimentell nunmehr verschiedene Instrumente und Stimmen und schließlich Vokale. Die komplexe Frage ist somit, wie die Sirene die Erforschung der klanglichen Dimensionen der Stimme aufruft, aber selbst noch an der Sprengung des damit einhergehenden experimentellen Rahmens einen immensen Anteil haben wird.

\section{Entwurf der Sirene}

Baron Charles Cagniard de la Tour, der von seiner Erfindung der Sirene erstmals 1819 in den Annales de Chimie et de Physique berichtet, 
erklärt im einleitenden Passus ein Steuerungsprinzip geradewegs zum physikalischen Wirkungsprinzip selbst:

Wenn der von den Instrumenten erzeugte Klang grundsätzlich von der regelmäßigen Folge vervielfachter Stöße abhängt, den sie an die atmosphärische Luft durch ihre Schwingungen abgeben, wovon die Physiker überzeugt sind, scheint es selbstverständlich anzunehmen, daß mit der Hilfe eines Mechanismus, der so zusammengesetzt ist, daß er die Luft mit derselben Geschwindigkeit und mit derselben Regelmäßigkeit anschlägt, ein Klang hervorgerufen wird [Cagniard de la Tour 1819, 167-168]. ${ }^{4}$

Es sind folglich nicht Schwingungen, die den Klang ursächlich hervorbringen, sondern eine Reihe von Stößen. Schwingungen, wie sie etwa von Saiteninstrumenten herrühren, nimmt die Luft nur in der Form einer Reihe von Stößen auf. Das aber heißt, daß Musikinstrumente keineswegs mehr als grundlegende Mechanismen der Klangerzeugung anzusehen sind. Denn die Sirene tritt nunmehr als Mechanismus an, der alle notwendigen und hinreichenden Elemente zur Klangerzeugung zusammenbringt. Daß in Wirklichkeit der Natur immer eine Kontinuität zu unterstellen ist, wird damit fragwürdig. Nicht mehr zwingend ist die infinitesimale, schrittweise Annäherung der Zeichensysteme an unauflösliche Kontinuitäten, sondern zum Programm wird offensichtlich, die Diskontinuität diskreter Zeichensysteme wirkungsmäßig selbst in das Experimentalsystem einzulassen.

Die Elemente, die die Sirenen ausmachen, sind Serien von Lochungen. Sie verdichten Luft zu einem Strom, doch sie sind keineswegs nur operable Elemente. Sie bedienen auch Zeichenvorstellungen, indem sie die Möglichkeit eröffnen, als diskrete Zeichen beliebig gesetzt und gelesen zu werden. (Abb. 1 und Abb. 2) ${ }^{5}$ Cagniard erwähnt die Verwendung des Lochkartenprinzips zwar nicht, doch diagrammatisch listet er Zahlen von Lochungen und alte Tonsilben auf, was der Transponierung einer mittelalterlichen, auf der Stimme basierenden Codierung in eine völlig neue maschinelle gleichkommt. (Abb. 3) Es ist sicherlich keiner Koinzidenz, sondern einem generellen Umbruch geschuldet, daß mit der Lochkarte, die seit 1807 zur Steuerung von Jacquards Webstühlen massenweise im Umlauf ist, einschreibbare Maschinenabläufe gängig werden.

\footnotetext{
${ }^{4}$ Im Orginal heißt es: „Si le son produit par les instrumens est dû principalement, comme le croient les physiciens, à la suite régulière des chocs multipliés qu'ils donnent à l'air atmosphérique par leurs vibrations, il semble naturel de penser qu'au moyen d'un mécanisme qui serait combiné pour frapper l'air avec la même vitesse et la même régularité, on pourrait donner lieu à la production du son."

${ }^{5}$ Die Abbildungen finden sich am Ende des Artikels, S.112.
} 
Eindrucksvoll stellt Cagniard unter Beweis, daß sein Mechanismus nicht an eine spezifische Materialität gebunden ist. Cagniard taucht das Instrument unter Wasser und stellt fest, daß sie bei gleicher Konfiguration, aber nun bei strömenden Wasser, den gleichen Ton trifft — zudem einen Ton wie von einer "voix humaine" [Cagniard 1819, 168]. Als das Instrument die Eigenschaft aufweist, auch ,im Wasser zu klingen" [Cagniard 1819, 171], heißt sie — in jedem Wortsinn - ihrem Erfinder: Sirene. ${ }^{6}$ Die schwingenden Körper wechseln, der identifizierbare Klang - der menschlichen Stimme nach wie vor gleich — bleibt. Doch die "Chocs" [Cagniard 1819, 168], die die Sirene der Luft wie auch dem Wasser beibringt, bezeichnen reine Ereignisse. Die Sirene ist nunmehr eine Klangquelle, ohne ein Klangkörper zu sein. Sie ist ein System, das zunächst einzig den Beweis führt, mehr als nur ein Medium in exakt bestimmbare Zustände zu zwingen. Die Sirene spart damit erfolgreich den Platz aus, der seit dem 18. Jahrhundert mit dem Begriff des Äthers als beobachtbare Identität von Wirkungen bei alternativer Einschaltung differenter Körper belegt ist.

Zwar legt die Sirene ihr mechanisches Prinzip in bestechender Weise offen, doch sie verschiebt die Klangformation ins Unvorstellbare: Sie stellt Tatsachen und Vorgänge sicher und gibt alle erdenklichen Formen zu berechnen auf. Bei schwingenden Saiten konnte es noch um die Visualisierung von Schwingungen durch eine nachträgliche Sichtbarmachung per Analogiebildung gehen. Diese Möglichkeit schließen die transparenten Trägermedien der Sirene - Luft und Wasser - aus. Statt dessen werden Präformationen der Beschreibbarkeit und Berechenbarkeit durch Elemente der Aussparung vor Augen gestellt und uneinsehbare Vorgänge dennoch kodiert. Cagniards Sirene folgt damit in aller Stringenz einem mathematischen Programm, das auf Leonhard Euler zurückgeht.

Mit Euler bricht sich eine Mathematik Bahn, die aufzeigt, wie auf dem Papier mit Diskontinuitäten und Unstetigkeiten umzugehen und jeder auf Kontinuität gründenden Naturvorstellung vorzuhalten ist. Wenn aber Euler zum Entsetzen d'Alemberts den Ausnahmefall der „choc des corps" [Zitiert nach Siegert 2000, 300], der das Kontinuitätsgesetz unterläuft, zur Regel erklärt, dann löst er die modellierende Mathematik aus Evidenz- und Plausibilitätsbereichen der Physis. Vielmehr verspricht erst Eulers Programm, Klänge diesseits von Elementen und Materialei-

\footnotetext{
${ }^{6}$ Seit dem Mittelalter treten in den Epen Sirenen als Amphibien auf (worauf mich dankenswerter Weise Friedrich Kittler hinwies) — eine literarische Stoffanwandlung mit weitreichenden Folgen: Richard Wagners Vorspiel des Nibelungenrings, das Rheingold, hebt ja der Bühnenfiktion nach mit einem Gesang der drei Rheintöchter an, der unter Wasser statthat.
} 
genschaften der Instrumente zu berechnen und zu synthetisieren, d.h. auch Signale und Stimmen [Siegert 2000, 298]. Schon zu ganz anderen Zeiten finden sich mathematische Beschreibungen von Instrumenten zur Klangerzeugung, die nicht selten über musikalische Wahrnehmungs- und instrumentelle Machbarkeitsgrenzen hinausschießen. Um aber zuvor auf Klangmechanismen zu stoßen, die ihre Existenz mathematischen Fragen und Argumenten verdanken, muß man wohl bis zu Hippasos' fragmentarisch kolportierten und andeutungsreichen Versuchen zurückzugehen [Zhmud 1994, 192].

\section{Stimme/Apparat}

Ist der Klang von Cagniards Sirene der einer Stimme oder der eines Instruments? Wieweit reicht die Analogie, die Cagniard selbst zieht, wenn er mit einem Instrument eine menschliche Stimme vernimmt, die ihm „Sirene" heift? Der Unterschied von Instrument und Stimme ist schon der peripatetischen Schule mit ein Grund, in der Mathematik die Möglichkeit der Umfassung beider zu suchen [Neumaier 1986, 12]. Das Instrument, das der Geometrisierung und Proportionstheorie am meisten entgegenkommt, ist ohne Zweifel das Saiteninstrument. Doch ausgerechnet deshalb erwächst noch einer ersten immanenten Musiktheorie ein Mittel der Ablehnung und der Selbstbegründung. So verwirft Aristoxenos eine Theorie der Musik, die mit euklidischen Mitteln auf die Teilung des Kanons und damit einzig auf die Einteilung konkreter Saiten hinausliefe. Erstens, so Aristoxenos, sei eine harmonische Ordnung des Melos auf die Musikinstrumente nicht vollständig abzubilden. Denn wäre dem so, dann klänge ein Instrument, egal wie man es spielt, nie falsch, was offenkundig nicht der Fall ist [Aristoxenos 1868, 59-61]. ${ }^{7}$ Und zweitens vermag das Ohr immer schon mehr an Musik zu vernehmen, als eine einzelne Stimme oder ein einzelnes Instrument hervorbringen kann [Aristoxenos 1868, 20 und 29]. ${ }^{8}$ Sind äußere Intervallgrenzen durch die Klangquellen selber gesetzt, so werden Binnenintervalle der Geometrie unterworfen. Um aber unendlichen Intervallbildungen zu begegnen und statt dessen die Auswahl musikalischer Intervalle zu begründen, bietet Aristoxenos eine Analogie auf, die auf der Ordnung des Alphabets beruht: Denn es zeigt sich, daß „nicht durch jede Art von Zusammensetzung der Buchstaben

\footnotetext{
${ }^{7}$ Zum Primat des Melos siehe [Busch 1998, 128]. Zum Problem der theoretischen Beschränkung allein auf Saiteninstrumente [Neumaier 1986, 119 und 144].

${ }^{8}$ Der Knabe etwa vermag den Gesang des Mannes genauso gut zu hören, wie dieser ihn, aber stimmlich-musikalisch trennt sie eine Oktave. Analoges gilt für das Verhältnis von Jungfrauenflöten und langen Flöten.
} 
[... ] eine Sylbe" entsteht [Aristoxenos 1868, 53]. Um es kurz zu machen, es sind unterschiedliche Praktiken der Musik, die pythagoreische Einheit von Mathematik und Musik in unterschiedliche Praktiken der Notation aufbrechen.

Gerade die Inwendigkeit und Unsichtbarkeit des Stimmapparates erweitert die Exteriorität der Zeichensysteme, während Instrumente, ob ihrer abzählbaren Elemente wie Löchern, Saiten und/oder Stegen, immer schon Registern, Merkmalen und Einteilungen unterliegen. Doch über Epochen sind Berechnung von Instrumenten und Führung von Stimmen unterschiedlichen Zeichenoperationen unterstellt. Guido von Arezzos Antiphonal, das die Vier-Linien-Notation der Partitur begründet, wendet sich ausdrücklich zuallererst an die Stimmführung und nicht an Instrumente. Mehr noch zielt sein Notationssystem gerade darauf, Instrumente abzulösen, die bis dahin der Singstimme im Zweifelsfalle Halt geben [Oesch 1954, 5-6].

Klangkörper indes zeigen mathematisch analysierbare Formen immer unterhalb der Schwelle anhaltender oder geräuscharmer Klänge. Das gilt für Mersennes Monochords mit extrem schweren und langen Saiten wie man sie vorher nur im Tennisspiel anzuwenden wußte [Dostrovsky \& Cannon 1987, 31] -, ebenso für Robert Hookes Rad mit schlagenden Stiften [Dostrovsky \& Cannon 1987, 32] und schließlich auch für Daniel Bernoullis und Eulers hängende Kette [Dostrovsky \& Cannon 1987, 5459] — obschon letztere über die Bestimmung absoluter Tonhöhen weit hinausführen und der Visualisierung von Teilschwingungen gelten. Allein Taylors Verwendung des Hemmungsrades einer Zimmeruhr in Verbindung mit dem „Hookeschen Rad“ ist der Versuch, die Generierung eines gleich bleibenden Klangs durch Bewegung diskreter Elemente konsequent vorzuschreiben [Dostrovsky \& Cannon 1987, 35].

Nur um den Preis, die Ordnung von Musikinstrumenten völlig unangetastet zu lassen, gelingt es um 1800 Tonfolgen automatisch aufzurufen und/oder aufzuzeichnen [Klotz 2000, 316-325]. Maschinen bedienen nun die ehedem anthropozentrisch ausgerichteten Schnittstellen von Maschinen. Dazu verkoppeln per Stiftwalzen und später per Lochkarten gesteuerte Musikapparate Maschinen mit eineindeutigen Zeichensystemen und machen bestimmte Klangereignisse erstmalig zu reversiblen Prozessen. Doch ausnahmslos sitzen Maschinen den gegebenen Registern und Klaviaturen anderer Maschinen auf. Von Kempelens Sprechmaschine schafft zwar darüber hinaus den Schritt, konsonantische Laute zu fixieren, ohne auf die präparierte Glottis und den Pharynx von Leichen zurückzugreifen wie Anatomen vor ihm. Seine Sprechmaschine setzt sich statt dessen aus Musikinstrumententeilen von Dudelsackpfeifen und gedeck- 
ten Oboen zusammen [Siegert 2000, 304]. Doch die Ausgefallenheit der Musikinstrumente, die von Kempelen verwendet, läßt nur den Schluß zu, daß er einzig blanke Empirie und nicht Zeichensysteme bemüht. Folgt von Kempelen mit seiner Sprechmaschine nur der Möglichkeit, die Euler mathematisch postuliert [Siegert 2000, 304-305], so schlägt Cagniard die Brücke zur Strenge mathematischer Modellierbarkeit. Der Unterschied ist fundamental: Stimmapparate anatomisch zu isolieren und dann $\mathrm{zu}$ reanimieren oder aber gegebene Instrumente auf die Nähe ihres Klanges zur Stimme abzuhören, ist eine Sache. Eine völlig andere ist es, ein System zu entwickeln, das seiner Form nach nicht mehr die geringste Ähnlichkeit zum Stimmapparat aufweist. Wenn schon das Rad kulturgeschichtliche Vorstellungen unhaltbar macht, daß alle Instrumente nur Extensionen und Projektionen von Körperorganen sind, dann führt der Einsatz des Rotationsprinzips erst recht von physiologisch homologen Strukturen unumkehrbar ab [Kittler 2000, 208]. Die Sirene generiert einen hörbaren Ton nicht vor Erreichen einer bestimmten Rotationsgeschwindigkeit. Kein Anschlag, wie bei einer zurückschnellenden Saite, garantiert von der ersten Bewegung an Klänge, sondern erst dezidierte Systemeigenschaften wie Umdrehungszahlen und Luftstromgeschwindigkeit. Die Sirene ist das erste Instrument, das musikalische Klänge von beliebiger Dauer, ungeahntem Oktavenumfang und Amplitudenumfang durch exakt einstellbare Lochungen hervorbringt. Sie ist überhaupt das erste Instrument, das wie eine „voix humaine“ klingt, durchstimmbar ist und dennoch nicht im Orchester der abendländischen Musik einen Platz findet. Erst die experimentelle Musik Edgar Varèses wird Sirenenklänge nach Helmholtz-Lektüren und dem Flohmarktfund zweier Handsirenen nach dem Ersten Weltkrieg erstmalig aufnehmen [de la Motte-Haber, 48].

\section{Klang/Maschine}

Wohl kein Zufall ist, daß der Tonumfang von Cagniards Sirene im Vergleich zu anderen Klangquellen in ganz unerhörte Dimensionen vorstößt. Cagniard schließt seinen Bericht damit, die Sirene produziere reinste Töne noch jenseits vom Tonumfang jener Klaviere, die sechs Oktaven umfassen [Cagniard 1819, 171]. Cagniard entwickelt die Sirene in einer Epoche ökonomischer und energetischer Maschinentheorien. Er wurde nobilitiert, nachdem er das königliche Spital und die königliche Werkstatt mit einer Gasbeleuchtung ausgestattet hatte [Payen 1971, 8-10]. Zuvor durchlief er die Pariser École polytechnique, verdankt seine Karriere also einer institutionellen Neugründung, die auf den Republikaner erster 
Stunde, Staatslenker, Militärstrategen und Maschinentheoretiker Lazare Carnot zurückgeht. Nicht wenige Überlegungen und Einlassungen Carnots richten sich auf die Maximierung und größtmögliche Ausbeutung von Kräften. In einer Schrift - die anhand eines neuen Explosionsmotors der Brüder Nièpce mit dem programmatischen Namen Pyréolophore dem generellen Krafterzeugung durch Maschinen nachgeht ${ }^{9}$ — stellt Carnot fest, daß die Antike nur Kräfte einzusetzen und umzulenken wußte, die längst als Wind, Wasser, und Feuer in Erscheinung getreten sind oder durch Sklaven und Tiere aufgebracht werden. Doch selbst unter Ausnutzung aller Hebel- und Zugvorrichtungen können die schon vorhandenen Kräfte bestenfalls effizient genutzt, nicht aber gesteigert werden. Carnot ging es aber um Verfahren der Freisetzung und Transformation von Kräften, die allein den Schlüssel zur unbekannten Steigerungsfähigkeit liefern [Carnot \& Berthollet 1806, 465].

Cagniard leitet zehn Jahre vor der Sirene mit einer anderen Maschine maßgeblich die Anfänge der Theorie der Thermodynamik ein. Als inverse Archimedes-Schraube ist sie in die Annalen eingegangen. Auch diese Maschine zeichnet wieder die stupende Eleganz aus, mit geradezu wenigen aristotelischen Elementen auszukommen und verschiedenste Materialkoeffizienten einzusparen. Sie steht in einer Reihe mit Maschinen wie der der Brüder Nièpce, die durch Ausdehnungen und Komprimierung von Luft angetrieben sind. ${ }^{10}$ Anderen ,air engines" hat sie voraus, schlicht elementarer beschaffen zu sein: ,it has no pistons, valves, and mechanical linkages." [Kuhn 1961, 572] Auch sie ist wie die Sirene eine in allen Teilen weitgehend berechenbare Maschine für Zustandsänderungen eines Mediums. Anstatt Wasser über einen Wasserspiegel hinaus zu befördern, zieht sie Luft ins Wasser hinein. Die Luft durchläuft dann Bereiche im Wasser von unterschiedlicher Temperatur. Nach der Anwendung der Maschine $\mathrm{zu}$ fragen, ist deshalb so nutzlos wie die Maschine für sich genommen: Sie stellt jedoch fünfmal so viel Kraft über eine Welle zur Verfügung, wie für den Antrieb der inversen Archimedes-Schraube benötigt wird. ${ }^{11}$

Cagniard kann dem geschlossenen Regelkreis aus Luft und Wasser mit einem Temperaturgefälle ablesen, daß es einen „kritischen Punkt" gibt — den Begriff hat Cagniard bei der Gelegenheit geprägt -, der ein

\footnotetext{
${ }^{9}$ Der Explosionsmotor und die Sirene weisen strukturelle Übereinstimmungen auf und zwar hinsichtlich der Mechanik, die die Kompression und die ihr abzuschöpfende Kraft auch möglichst effizient für die eigene Steuerung einsetzt.

${ }^{10}$ Laut Thomas Kuhn, ,seems [Air] to have been the principal alternate working substance seriously considered by engineers during the early decades of the nineteenth century." [Kuhn 1961, 570]

${ }^{11}$ Die Energie zur Aufrechterhaltung der unterschiedlichen Temperaturen wird allerdings nicht beziffert.
} 
Element in einen anderen Zustand übergehen läßt. Carnots Sohn Sadi bestimmt in Anschluß an Cagniard den theoretisch höchsten Wirkungsgrad zweier Temperaturen. Thomas Kuhn - bevor er generell von Paradigmenwechseln und Strukturen wissenschaftlicher Revolutionen schreibt weist in einer gesonderten Untersuchung Cagniards Maschine als Basis von Carnots Theorie der Krafterhaltung aus [Kuhn 1961]. Ließ sich auf Archimedes' Wissen um die Hydraulik immerhin bestimme Staatsökonomien gründen, so kennen Cagniards Maschinen keine unmittelbare Anwendbarkeit und eben darin liegt ihre Stärke. Die französischen Theoretiker hatten angelsächsische Praktiker der Dampfmaschinen vor Augen und wußten, daß sie gegen diese auf dem Feld konkreter technischer Lösungen kaum Land hätten gewinnen können. Ihr Igel im Rennen mit dem Hasen sind mathematische Argumente, die mit sprunghaften $\mathrm{Zu}-$ standsänderungen, Nicht-Identitäten und Differenzen rechnen, um prinzipielle Grenzen aufzuspüren. Wohl kein Zufall ist, daß die Theoretiker von „kritischen Punkten“ und höchsten Wirkungsgraden gleichzeitig und vielleicht gar nicht so anders - in Frankreichs revolutionären Zeiten in höchsten Ämtern Staatsgeschäfte regelten. Sie bringen Maschinen und Staatsformen hervor, die hochgradig epistemisch sind, also zwischen Diskursen und Praktiken ihren Einsatz finden.

\section{Ohms „Gehörstäuschungen“"}

Cagniards Maschinenentwurf der Sirene platzt überraschend in das Feld akustischer Forschungen hinein. Sie geht aus Diskursen hervor, die nicht zunächst das Feld der Akustik behandeln, sondern die auf die Entfesselung von Energien und Dynamiken gerichtet sind. Vor diesem Hintergrund behauptet sich die Sirene als Referenzsystem für die Akustik und steht am Ende einer langen Debatte, intervallische und damit relative Klangbeziehungen in die Schranken der absoluten Tonhöhenbestimmung zu weisen. Cagniard knüpft deshalb mit dem Meßsystem der Sirene an Joseph Sauveurs Fundierung absoluter Tonhöhen an, das immer noch als letzter Stand der Dinge gilt [Cagniard 1819, 170]. Bei Sauveurs Verfahren absoluter Tonhöhenmessung ist die Wahrnehmung konstitutiv: vorausgesetzt wird ein musikalisches Gehör, das erstens den Halbtonschritt zwischen zwei Tönen sicherstellt und zweitens die dann im weiteren auftretenden Schwebungen ohne Schwierigkeiten mitzählen kann. Die absolute Frequenz der Tonhöhe beider Töne läßt sich dann mit ziemlicher Genauigkeit angeben [Dostrovsky \& Cannon 1987, 33-35]. Da Cagniards Sirene eine beliebig isochron aufgerufene Anzahl an Impulsen festschreibt und registriert, ist der Nachweis einer Schwingungsanzahl erst gar nicht zu 
führen. Ein Verfahren sicherte ehedem die Reproduzierbarkeit des Experiments, indem es qua Vorschriften Wahrnehmungsakte auf den Klang lenkte. Nun aber garantiert ein System Reproduzierbarkeit, in dem es die Wahrnehmung sowohl auf den Klang, als auch auf die Zeichen seiner Kodierung ausrichtet. Das aber heißt, daß visuelle Zeichenkonfigurationen der Sirene den gleichen Raum einer Prüfung einnehmen, wie die erzielten akustischen Effekte. Und in der Tat, es dauert nicht lange und die Sirene erschüttert die in der Akustik tief verankerte Vorstellung des Isochronismus fundamental und damit das Wissen vom Klang überhaupt.

Im Disput zwischen August Seebeck und Georg Simon Ohm sind Spuren der Erschütterung durch Sirenenexperimente nachzulesen. Mehrfach ist die Auseinandersetzung in der Forschungsliteratur aufgegriffen worden [Zuletzt Turner 1977; Vogel 1993], wohl nicht zuletzt weil Helmholtz darin einen Anfang machte [Helmholtz 1856, 263 und 289-290]. Es wird deshalb hier mehr als anderorts eine Lesart aufgenommen, die Helmholtz' sich darauf entwickelndes epistemologisches Modell an die Seebeck-Ohm Kontroverse zurückbindet. Dann läßt sich die These erhärten, daß diskursiv randständige Elemente, die die wissenschaftliche Verständigung stören und verunsichern, im Zentrum einer nachmaligen Erkenntnistheorie auftauchen. Denn Helmholtz differenziert schließlich im Bereich des Klanges Ebenen aus, die vor allem Disziplinen wie Physik, Physiologie und Psychologie und ihre Unterscheidung re-konstituieren. Deutlicher soll damit werden, warum von einem historischen Moment an - der sich mit Hermann von Helmholtz' fachlich weitreichenden Arbeiten abzeichnet - an fachspezifischen Disziplinen kein Weg vorbeigeht und keine Disziplin ohne jede andere auskäme.

Nahezu ohne Vorarbeiten und Vorlauf kommt Georg Simon Ohm darauf, eine „Definition des Tones, nebst daran geknüpfter Theorie der Sirene" anzugeben. Hervorgetan hat sich Ohm bekanntlich mit seinen Arbeiten auf dem Feld der Elektrizität. Im Kern allerdings zeichnen alle seine Schriften mathematische Argumente aus, wie sie um diese Zeit alles andere als selbstverständlich sind. Georg Simon Ohm gehört zu den ersten und wenigen deutschen Experimentalphysikern, die die Schriften des wissenschaftlich-mathematischen Stabs um Carnot verfolgten, bevor diese selbst in Frankreich an Wirkung verlieren [Heidelberger 1979, 135]. Ohms Arbeit über die galvanische Kette lehnt sich eng an Joseph Fouriers Théorie analytique de la Chaleur an [Heidelberger 1979, 100 und 134]. Ohm schlägt damit eine völlig andere Richtung ein, als Johann Wilhelm Ritters galvanische Versuche sie vorgaben. Ritters Versuche, um nicht zu sagen Selbstversuche, da er keine seiner Körperöffnung ausließ - darunter Augen, Mund und hohle Zähne, um von anderen ganz zu schweigen 
-, erklären Wirkungen des Galvanismus sensuell. Erst Ohm liest ziemlich gegensätzlich an der galvanischen Kette abgenommene Meßwerte jenes berühmte mathematisch einfache Verhältnis ab, wonach „die Wirkung einer [galvanischen] Kette gleich ist der Summe der elektromotorischen Kräfte geteilt durch die Summe der Widerstände." [Zitiert nach Füchtbauer 1947, 256]. Es verstreichen aber zwei Jahrzehnte, um ein Feld von Anwendungen zu schaffen, innerhalb dessen Ohms entscheidende Größen zu stabilisieren zur Notwendigkeit wird. Aufmerksamkeit erlangt Ohm denn erst mit dem Aufkommen elektrischer Telegraphen [Heidelberger 1979, 141-146]. Ohm nutzt die Aufmerksamkeit um ein weitere Formel aufzustellen, die in der Physiologie bis heute ebenfalls als Ohmsches Gesetz bekannt ist: Für die Bildung eines Tons mit der Schwingungsmenge $m$ ist vorauszusetzen, daß bei ihr nur Eindrücke sinusförmiger Prägung zum Tragen kommen, die bei gleich bleibenden Schwingungsphasen in ein und denselben einfachen Verhältnissen zueinander stehen und durch eine bestimmte Amplitude gekennzeichnet sind [Ohm 1843, 518]. Weder ist das Modell neu, Saitenschwingungen auf einfache, pendelartige Schwingungen zurückzuführen [Dostrovsky \& Cannon 1987, 24-27 und 66], noch die Annahme, komplexe Schwingungen einer Saite - komplex schon deswegen, weil niemals nur ein Ton ohne Obertöne von ihnen auszugehen scheint -, könnten als eine Überlagerung sinusförmiger Teilschwingungen geschrieben werden [Dostrovsky \& Cannon 1987, 53]. Letzteres fußt auf einem rein funktionstheoretischen Ansatz Daniel Bernoullis, die Lösung einer Variable der zweidimensionalen Wellengleichung d'Alemberts anstelle der üblichen Differentialgleichungen in Form einer trigonometrischen Reihe anzugeben [Dostrovsky \& Cannon 1987, 68; Grattan-Guiness 1969, 231]. Bernoullis Lösungsweg markiert einen Endpunkt innerhalb der Mathematisierung der klingenden Saite. Er führt zu dem double bind: daß die Bewegung der Saite nicht einfach schlicht als eine einzige sinusförmige aufzufassen ist, zeigt gerade ihre mathematische Zerlegung in ebensolche Sinus- und Kosinusausdrücke. Doch kein materieller Träger außer dem Papier weist die gleichmäßigen Kurvenverläufe einzelner Komponenten der Funktionstheorie auf. Wohl nicht ohne Grund führt erst Joseph Fourier elementare Winkelfunktionen auf dem ganz anderen Feld der Wärmeleiter, das per se einer unmittelbaren Sichtbarkeit entzogen ist, systematisch und seitenweise in Serien aus - sehr zum Staunen und Schrecken der mathematischen Mitglieder der Académie des Sciences [Grattan-Guiness 1969, 230-231]. Mochte Ohm schon im Zuge seiner Arbeiten zur galvanischen Kette auf Fouriers Methode gestoßen sein, von ihr Gebrauch machte er erst, um die Reihenentwicklungen nach fast hundertjähriger Vakanz wieder ins Zentrum 
der Akustik zurückzuführen:

Als Mittel der Beurteilung, ob in einem gegebenen Eindruck die Form $a * \sin 2 \pi(m t+p)$ als reeller Bestandteil enthalten sey oder nicht, gebrauche ich das durch seine vielfachen und wichtigen Anwendungen berühmt gewordene Theorem von Fourier [...]. [Ohm 1843, 519] ${ }^{12}$

Ausgelöst wird Ohms Intervention durch die experimentelle Eröffnung Cagniards, Seebecks und anderer, daß Sirenen Schwingungsvorgänge zerlegen. In gewisser Weise holen Sirenenexperimente damit eine mathematische Entwicklung ein, die mittels Fourieranalysen über partielle Differentialgleichungen hinausgeht und zu einem Funktionsbegriff führt, der vor Diskontinuitäten nicht halt macht [Siegert 2000, 296-299].

Seebecks Sirenenexperimente offenbaren, daß äquidistant gesetzte Lochungen und in gewissen Grenzen um den gleichen Mittelwert variierende, angeblasen, zu gleichen Höreindrücken der Tonhöhe führen. Synchrones Anblasen einer Reihe von Lochungen an sich gegenüberliegenden Stellen löscht den Klang vollständig aus. Impulse von Lochungen mit zwei unterschiedlichen Abstandmaßen lassen indes zwei Töne erklingen, die annähernd Sirenenkonfigurationen mit einem einzigen Abstandmaß von der Summe beider und deren Hälfte gleichkommen [Ohm 1843, 515517]. Die Sirene erreicht also auf ganz unterschiedliche Weise gleiche Tonhöheneindrücke. Um mit Unterscheidungsmerkmalen zurechtzukommen, die an der schwingenden Saite nicht zu beobachten und mit dem Gehör mitunter nicht zu unterscheiden sind, fordert Seebeck, die Klangbestimmungen an die Impulskodierungen der Sirene zu binden [Ohm 1843, 514]. Damit taucht die Schwierigkeit auf, Phänomene losgelöst vom Experimentalsystem ihrer Hervorbringung zu denken und zu beschreiben. Von den Experimentalsystemen des 20. Jahrhunderts wird es schließlich heißen können:

Naturwissenschaftler denken, begrenzt durch den hybriden Kontext ihres jeweiligen Experimentalsystems, in den Koordinaten solcher Räume möglicher Darstellung. Genauer gesagt, sie spannen solche Repräsentationsräume auf, indem sie ihre Grapheme zu epistemischen Dingen verketten. [Rheinberger 2001, 8-9].

Der Disput, der sich zwischen Seebeck und Ohm sich zuträgt, bildet indes den Prozeß der Hybridisation selbst ab. Im Zuge der Hybridisation vermitteln und repräsentieren Grapheme und Zeichensysteme nicht mehr

\footnotetext{
${ }^{12}$ Die Schwingungsmenge oder modern gesagt Frequenz ist dabei $m, t$ die Zeit, $a$ die Schwingungsweite und $p$ die Schwingungsphase.
} 
allein experimentelle Befunde, sondern vermögen sie zuallererst hervorzubringen.

Bei dem Disput nimmt Ohm vordergründig die Position ein, „die Definition des Tones, wie sie von unseren Vorfahren auf uns übergegangen ist" [Ohm 1843, 517], nicht vorschnell verwerfen zu wollen. Stützten sich vormalige Definitionen des Tones auf die Evidenz des Eindrucks einer Schwingung und einer Tonhöhe, dann führt Ohms Bestreben, an der Relation festzuhalten, zur Annahme beliebiger einfacher Teilschwingungen. Damit ist nicht mehr ausgeschlossen, daß unterschiedlich zusammengesetzte Teilschwingungen den Eindruck der gleichen Tonhöhe hervorbringen. Doch Ohm erweitert nicht etwa nur eine überlieferte Theorie, um sie zu bewahren. Denn was er dabei unter der Hand einführt, ist so radikal wie neu: Als einziges Experimentierfeld bleibt dafür der Evidenzbereich des Zeichensystems. Jeder Klang und zwar ganz egal welche visuellen Evidenz seine Erzeugung bietet, hat sich nunmehr mathematischen Gleichungen zu fügen.

Man kann die Auseinandersetzung zwischen Ohm und Seebeck deshalb auf unterschiedliche Effekte von Klang- und Zeichensystemen zurückführen, die Wahrnehmungen dissimilieren. Der Einladung Seebecks, ihn in Dresden zu besuchen, und der Hoffnung, er werde sich „nicht die Ohren vor den Gesängen [seiner] Sirene verstopfen" [Zitiert nach Turner 1977, 22, Fußnote 21], kommt Ohm gar nicht erst nach. Obwohl oder gerade weil Ohm sich mit einem fundamentalen Gesetz in die Geschichte der Hörphysiologie eingeschrieben hat, kann er doch von sich selbst behaupten, daß er über Klänge „fast wie ein Blinder von der Farbe rede" [Ohm 1844, 17]. Im Disput mit Seebeck fürchtet Ohm nicht die Klänge neuer Klanginstrumente, sondern „Druck, Schreib- und auch wohl Gedankenfehler" [Ohm 1844, 5]. Ohms Einlassungen gründen allein auf Beschreibung von Sirenenexperimenten, die Seebeck unternahm, die bemerkenswerter Weise als deskriptiv hinreichend angesehen werden und eigene Experimente nicht mehr als notwendig erscheinen lassen. Poggendorffs Annalen der Physik, die Zeitschrift, in der Seebeck und Ohm Beschreibungen ihrer Untersuchungen einschalten, werden selbst zum Schauplatz widerstreitender Experimentalsysteme, indem sie eine aktive Funktion übernehmen. Die Zeitschrifteneinschaltungen beanspruchen, Experimentalsysteme und Experimente in Zeichen der Rede und Rechnens zu überführen und umgekehrt Rechnungen und Vorschriften in Experimentalsysteme einzuspeisen. Allerdings kennen diese Praktiken keine Theorie. Sichtbar werden sie deshalb nur, wenn sie versagen. Im Fall von Seebeck und Ohm gehen die Praktiken an der neuralgischsten Stelle fehl. 
Sie führen Ohm zur falschen Annahme, daß die Sirene einen Sinusgenerator darstellt, wie ihn die Fourieranalyse impliziert. Denn Ohm geht bis zu einer Richtigstellung Seebecks davon aus, daß im Falle äquidistanter Lochungen der Sirene keine „Beitöne“, also Obertöne auftreten:

Ich war berechtigt diesen Satz so bestimmt auszusprechen, weil ich voraussetzen durfte, daß Seebeck, ein so sorgsamer Beobachter, einen so wichtigen Umstand nicht verschwiegen hätte, hätte er ihn wahrgenommen; und doch schloß ich fehl, weil Seebeck die Beitöne zwar beobachtet hatte, aber diese Wahrnehmung nicht veröffentlichte, weil er damals noch diese Töne als zufällig entstandene ansah. [Ohm 1844, 5, Fußnote 1]

Helmholtz' Hinweis, Ohm selber hätte die Notwendigkeit gefordert, Töne ohne Obertöne zu generieren [Helmholtz 1856, 267], verwechselt also nur die Verwechslung Ohms mit seinem eigenen Forschungsprogramm. Das aber heißt, einer mathematischen Methode der Fourieranalyse, die sich längst etabliert hat, die sogar Seebeck und dann Helmholtz von Ohm sofort übernehmen, eine Begründung nachzuliefern. Denn schließlich unternimmt es erst Helmholtz, eintönige Anteile im Obertonspektrum einer Stimmgabel durch einen Resonator zu isolieren [Helmholtz 1856, 267-268]. Damit schafft Helmholtz erst den Schritt, auch instrumentell komplexe Klänge aus einzelnen Tönen wieder zu resynthetisieren. Selbst wenn Helmholtz mit Stimmgabeln operiert, bleibt die Sirene das Referenzmodell. Denn die isolierten Sinustöne sind Obertöne. Sie stehen nicht mehr in unmittelbaren Bezug zum Instrument, sondern schon zu einem Schallereignis, dem Grundton. Tatsächlich schlägt Helmholtz im Bereich der tonalen Autoreferenzialität - der Evozierung und Verstärkung von Tönen durch Töne - die Brücke zur Genierung von Sprachlauten und ruft allgemein die instrumentelle Beherrschung von Klangfarben auf den Plan.

Helmholtz verdankt, so steht es zu vermuten, der Kontroverse zwischen Ohm und Seebeck aber mehr noch als nur Desiderate, die er den Mißverständnissen abliest. Ohms Rettungsversuch seiner durch Seebeck in Kritik geratenen schriftlichen Ausführungen wird bei Helmholtz als experimentelle Methode wiederkehren, die Zeichensysteme in Experimentalsysteme integriert. Der Experimentalphysiker Ohm gibt denn seinen fragwürdig gewordenen Gleichungen den Status eines mathematischen Experiments, dessen Scheitern wiederum nur durch einen apparativen Versuch aufzudecken ist. Das einzige nicht papierene Experiment, das Ohm unternimmt, zielt also erst gar nicht auf akustische, sondern auf graphische Klärung. Ohms Versuch hat einzig zu demonstrieren, daß ein sinusförmiger Schwingungsvorgang an die Stelle eines Impulses zu 
setzten ist. Operierte Cagniard mit den durchsichtigen Medien Wasser und Luft, dann moduliert Ohm den Schein des Feuers. Die angeblasene Flamme einer Kerze reicht Ohm, um die Ordnung einer Natur wiederherzustellen, die seit Leibniz bekanntlich keine Sprünge macht. Denn die Flamme vollführt eine Bewegung, die über die Dauer eines kurzen Luftimpulses hinausgeht und auch in die Richtung des unterbrochenen Luftstroms zurückschlägt [Ohm 1843, 564-565]. Ohm nimmt sein eigenes Experiment und die Befunde von Seebeck zur Verteidigung seiner mathematischen Synthesen in Anspruch, deren beliebige Freiheitsgerade durch Visualisierungstechniken erst einzuschränken sind. Indem die mathematischen Synthesen jeden noch so komplexen Schwingungsverlauf darstellen können, ist die Beweislast nunmehr eine umgekehrte. Es gilt Verfahren sicherzustellen, die aus einer Menge unendlicher mathematischer Möglichkeiten eine Synthese herausstellen, die mit bestimmten Beobachtungen von Phänomen in Einklang ist:

Es konnte mir nicht entgehen, daß die aus meinen besonderen Formen sich für die einzelnen Töne ergebenden Intensitäten mit der Erfahrung nicht wohl zusammenstimmen, insbesondere weil ich voraussetzte und auch [...] kategorisch ausspreche, daß Seebeck in solchen Fällen, wo die Löcher gleich weit von einander abstanden, nie einen der höheren Töne beobachtet hat. Jedoch hatte ich keine Ursache, mich hierüber zu wundern, da die Besonderheit der meinen Formeln zu Grunde liegenden Eindrücke, die offenbar denen in der Wirklichkeit auch nicht von ferne gleichen konnten, mir gerade bei einer wirklichen Auswerthung der Formeln nicht viel Richtiges zu geben versprach. Dessen ungeachtet war ich begierig zu sehen, wohin mich eine fortgesetzte Vergleichung derselben mit der Erfahrung führen würde. So wurde ich bis dahin geführt, daß diese besonderen Formen in Seebeck's Versuchen von jedem einzelnen Eindruck, verlangen, daß er sich über das ganze Intervall erstreckt habe, und der [...] besprochene Versuch zeigte zwar, daß diese Folgerung nicht unrichtig sey, zugleich aber auch auf eine unzweideutige Weise, daß die Sireneneindrücke entschieden doppelseitig sind, daß also die Voraussetzung, von welcher ich ausgegangen war, unrichtig ist, durch welchen Ausgang meine Neugierde vollständig befriedigt worden war. [Ohm 1844, 5-6]

Nicht nur läßt sich Ohm von „Formeln“ in „fortgesetzter Vergleichung derselben mit der Erfahrung" führen, sondern sie bringen ihn dazu, auch befreundete Musiker zu Wahrnehmungsexperimenten anzuleiten. Doch algebraische Gleichungen zeigen Lösungen von einer Unverrückbarkeit, die der Wahrnehmung nicht zukommt. Denn erst Ohms definitorischmathematische Postulate lassen Seebeck konträre Beobachtungen feststellen, die ihm zuvor entgangen waren. Für Ohm jedenfalls fallen Sinnesorgane damit als untrüglicher Referent von Versuchen aus: 
Ehe ich aber zu solchen Annahmen [nämlich die Seebecks], die mir unaufhörlich ihr geisterhaftes „Ich bin's und bin's auch wieder nicht" schmerzhaft in das Ohr raunen, meine Zuflucht nehme, stell ich lieber, keck wie Columbus, das Ei gleich auf die Spitze und behaupte: Jene von Seebeck wahrgenommenen Widersprüche beruhen auf einer Gehörstäuschung, in welcher unser Ohr befangen ist, auf ähnliche Weise, wie unser Auge in einer Gesichtstäuschung da befangen ist, wo es eine Mitteltinte an dunkler Gränze für heller, an heller Gränze für dunkler hält, als sie wirklich ist. [Ohm 1844, $15]$

Auf Ohms Rechenpapier zeichnet sich in beunruhigender Weise eine Wirklichkeit ab, die „Täuschungen“" und „Verwöhnungen“" [Ohm 1844, 17] erst offenbart: Unter anderem ordne das Ohr „unbewusst" Obertöne in harmonischen Verhältnis dem Hauptton zu, so daß es den „Hauptton für stärker ansieht, als er wirklich ist, und seine Beitöne für schwächer, als sie wirklich sind." $[\text { Ohm 1844, 15 }]^{13}$ Die medialen Differenzen, die die Auseinandersetzung zwischen Seebeck und Ohm antreibt, werden schließlich genau die Ebenen aufspannen, auf denen Helmholtz seine Erkenntnistheorie ausrichtet.

\section{Helmholtz' Experimente am „Selbstbewusstsein“}

Der Perzeption hat man seit Leibniz bis zu Kant kaum mehr Rechenfähigkeit zugetraut als aus lauter einzelnen Ereignissen die Summe [Leibniz 1765, 47] oder allenfalls die „Proportion der Zeiteintheilung“ [Kant 1790, $324 \S 51]$ zu ziehen, die apperzeptiv nur als einheitlicher Klang der Reflexion und Beurteilung zugänglich ist. Immerhin werden Sinnesorgane damit selbst zum Schauplatz der Mathesis elementarer Rechnungsarten. Doch Helmholtz wird einmal den gesamten Streit zwischen Seebeck und Ohm auf mangelnde Unterscheidung von Perzeption und Apperzeption zurückführen können, da Wahrnehmungstechniken nicht bereitstanden, d.h. Techniken zur Wahrnehmungslenkung als auch Technologien zur Wahrnehmungsermöglichung wie Resonatoren [Helmholtz 1913,

\footnotetext{
${ }^{13}$ Die Geister, die neue mediale Konfigurationen riefen, werden aber erst Medien austreiben. Denn erst mit der Entwicklung der Telefons stieß man wieder auf Seebecks "Geisterton" und entdeckte seine Arbeiten aufs neue als Residuumtheorie. Technisch bedingt übertrugen Telefone gewisse untere Spektren des Sprachsignals nicht. Obwohl damit klar ist, daß die Grundfrequenz des Sprachsignals, durch die sich etwa Männer- und Frauenstimmen unterscheiden, nicht übertragen wird, ist sie deutlich zu vernehmen und in ihrer Tonhöhe zu bestimmen [Autrum 1976, 496]. Darüber hinaus bildet das Verständnis von Frequenzgruppen und kritische Bandbreiten, die auf verschiedensten Weisen angeregt zu ähnlichen oder nicht mehr zu unterscheidenden Höreindrücken führen, die Grundlage für heutige Industriestandards von Audioformaten, die von Psychoakustik Gebrauch machen.
} 
107-108]. ${ }^{14}$ Doch Ohm und Seebeck gehören da eventuell schon einer anderen Epoche an. Denn jener Morgen am 22. Oktober 1850, an dem Gustav Theodor Fechner seiner Biographie nach länger als gewohnt im Bett blieb, läßt sich als Erwachen einer neuen Episteme ansehen. Sie kehrt das Innerste nach Außen und überführt das Einfachste in ungeahnte Komplexität. Fechner weist an der Haut nach, daß die Intensität einer Empfindung in einem reziprok logarithmischen Verhältnis zur Intensität des Reizes steht [Wundt 1901, 66-67]. Lange bevor der Entwurf einer analogen Maschine zur Berechnung von Logarithmen vorliegt übrigens Charles Babbages Initialtraum seiner Differential Engine -, ist sie gefunden: Mit Zirkeln lassen sich lineare Größen abtragen und logarithmische aufspüren.

Doch Fechner steht mit seinem Befund nicht allein da - es handelt sich bei ihm nicht um einen singulären Befund, sondern um ein weitreichendes epistemologisches Programm. Wilhelm Wundt findet, daß die Optomotorik des Menschenauges wie Gauss' neue und bahnbrechende Methode kleinster Quadrate zur Fehlerminimierung verfährt [Lenoir 1993, 137]. Daß Beobachtungsfehler mit Gauss' Verfahren reduziert werden können, heißt demnach nur eine intrinsische Blickstrategie fortzuführen und zu entfalten. Gerade weil die Analogie der mathematischmeßtechnischen Prüfung standhalten muß, zählen Analogien der Substanz und Form nach nicht mehr. Wundts Opthalmoskop hat äußerlich mit einem Augapfel wenig gemeinsam, sind doch beispielsweise an die Stelle von Muskeln Federn getreten, deren Kontraktionseigenschaften jedoch nach Maßgabe von Muskeln berechnet werden können [Lenoir 1993, 135]. Schließlich greift Helmholtz Ohms mathematischen Apparat der Fourieranalyse nicht allein als Methode auf, sondern erklärt ihn zur physiologischen Wirkungsweise selbst [Helmholtz 1913, 97]. Ist bei $\mathrm{Ohm}$ noch von einer "Suceccession von Eindrücken auf unser Ohr" [Ohm 1843, 497] die Rede und sind sonst klangliche Geltungsbereiche kaum differenziert, dann nimmt erst Helmholtz einen fundamentalen Wechsel vor und „Eindrücke der Ohren“ sind fortan als genetivus subiectivus zu lesen - gemeint sind nunmehr Eindrücke, wie sie Ohren empfinden und nicht mehr Eindrücke, die auf Ohren treffen. In Helmholtz' unscheinbarer Schrift „Ueber Combinationstöne" bahnt sich die Wende an, die in seinem umfassendem Buch Über die Lehre der Tonempfindungen als physiologische Grundlage für die Theorie der Musik als Positions-

\footnotetext{
${ }^{14}$ Die ersten Zeilen von Helmholtz „Die Lehre von den Tonempfindungen“ sind all denjenigen gewidmet, durch deren finanzielle Unterstützung neue Instrumente beschafft werden konnten, die über das Inventarium eines physiologischen Institut hinausgingen.
} 
wechsel bereits im Titel schließlich angezeigt ist. So entschieden damit zeichenhafte Operabilitäten physiologischen und mithin psychologischen Funktionsweisen zu entsprechen haben — womit Techniken zur Übertragung in den Bereich der Wahrnehmbarkeit entfallen, steht doch die Wahrnehmung selbst auf dem Prüfstand -, so wenig legt die Analogisierung die Richtung für einen Erkenntnisgewinn fest. Das freigelegte und präparierte Innenohr gibt Helmholtz Nervenbündel zu erkennen, die die Hypothese nahe legen, daßs sie jeweils Pendeln mit Schwingungseigenschaften von einzelnen Partialtönen gleichkommen [Vogel 1993, 267268]. Mit anderen Worten: Helmholtz geht davon aus, daß das Hörorgan an komplexen Klängen Fourieranalysen vornimmt [Helmholtz 1913, 97; Lenoir 1994, 197-198]. Der mathematischen Analyse folgt demnach die physiologische Analyse auf dem Fuß, aus der wiederum die Synthese in Form elektromechanischer Systeme hervorgehen kann. Denn Helmholtz' elektrisch angesteuerte und durch Resonatoren verstärkte Stimmgabeln demonstrieren im Umkehrschluß, daß die Zusammensetzung der einzelnen Partialtöne als ein komplexer Klang vor dem Ohr besteht. Im Fall gerade erst aufgekommener elektromechanischer Telegraphen schlagen die Analogiebildungen die andere Richtung ein. Für Helmholtz ist damit das Modell gegeben, die Wahrnehmung bei Beibehaltungen aller zeichentheoretischen Implikationen als einen Telegraphen zu begreifen [Lenoir 1994, 186].

Johannes Müllers These, daß spezifische Sinnesenergien Reize nicht ihrer Substanz nach vermitteln [Müller 1833-38, II/2, 260], erreicht in ihrer Verlängerung, daß die Wirkungsweise der Sinnesorgane losgelöst von einer konkreten Materialität angesehen wird. Um so mehr können Sinnesorgane damit als Problemstellung und Lösungsvorgabe eines mathematischen Programms figurieren. In dem Maße Lebenswelten und lebendige Organismen ein und denselben zeichenverhafteten Regelsystemen unterworfen werden, gewinnt es noch durch deren Wechselwirkungen an Aussagekraft. Hochgradig berechenbare Experimentalsysteme dringen in Lebenswelten ein, gehen von Ausnahme- in Normzustände über und erweisen damit Sinnesorgane als nicht weniger berechenbar. Während der Vitalismus zunehmend haltloser wird, weil spezifische Wirkungen sich nicht als unlöslich in Organismen verwurzelt erweisen, sondern ihre Übertragung auf andere Felder gerade zur experimentellen Maxime wird, ist schier mechanistischen Modellen um nichts weniger der Boden entzogen: Distanznahmen und Unterscheidungen von Objekt und Subjekt als tragenden Säulen vormaliger Experimente fallen. Vielmehr läßt Helmholtz keinen Zweifel daran, daß das Experiment, das er nur als 
den Sonderfall einer geschärften Wahrnehmung ausweist, ${ }^{15}$ gar nicht auf Distanzgewinnung und Objektivierung abzielt. Die Methode trigonometrischer Reihenentwicklung, die Fourier erstmalig in seiner Theorie der Kette anwandte, und Ohm dank analoger Untersuchung der galvanischen Kette dann auf das gänzlich andere Feld der Akustik zu übertragen weiß, schließst Helmholtz kurz: In Reihe oder Serien geschaltete Winkelfunktionen nehmen bei ihm einen Raum ein, der rechnende Subjekte und berechnete Objekte gleichermaßen durchquert und im Experiment verzahnt. Vor dem Hintergrund visueller Sinneseindrücke, die im Zuge von Bewegungen zustande kommen, begründet Helmholtz das Experiment in der Beobachtung von Wahrnehmungen unter wechselnden Einwirkungen, den das forschende Subjekt sich bewußt aussetzt:

Die überzeugende Kraft jedes Experiments ist aber hauptsächlich deshalb so sehr viel größer, als die der Beobachtung eines ohne unser Zuthun ablaufenden Vorganges, weil beim Experiment die Kette der Ursachen durch unser Selbstbewusstsein hindurchläuft. [Helmholtz 1879, 33]

Helmholtz' Epistemologie situiert ein Zeichensystem noch in den äußersten Sinnesorganen, die aus mannigfaltigen Reizen „induktive Schlüsse“ ziehen, im Zuge einer Aneignung, die dem Lernen einer Sprache gleichkommt [Helmholtz 1879, 12-13 und 26; Helmholtz 1896, 592]. Betreffen erdenkliche Modalitäten bei Kant allein Denkkategorien [Kant 1781, 5963], legen sie bei Helmholtz — der Johannes Müller darin folgt - die Sinne auf spezifische Empfindungsenergien und -weisen fest [Helmholtz 1879, 33; Helmholtz 1896, 594]. Ehedem als unüberwindlich konstituierte Schranken zwischen Perzeption und Apperzeption werden durchlässig und regelbar, soufflieren doch zeichen- und signalgebende Systeme wie zu allererst die Sirene dem Wissen ein Weg, der das Selbstbewußtsein in die mathematische Ordnungen des Unbewußten einläßt. War nach Kant alle transzendente Form, die jeden Gedanken begleiten können muß, schlicht geschenkt, dann läßt Helmholtz' Empirismus eine Wahrnehmung denkbar werden, die sich an der Empirie ausbildet [Helmholtz 1896, 602] ${ }^{16}$ und sich dann in ihrer eigenen Empirie selbst genug sein kann. So mag es zutreffen, konstatiert Helmholtz,

daß wir nach Aussage unseres Bewußtseins wenigstens ein Glied von der Kette der Ursachen, die zur Wahrnehmung führen, unseren Willensimpuls,

\footnotetext{
${ }^{15}$ Zur Strukturhomologie von Wahrnehmung und Experiment [Siegert 2000, 294].

${ }^{16}$,Wir könnten in einer Welt leben, in der jedes Atom von jedem anderen verschieden wäre, und wo es nichts Ruhendes gäbe. Da würde keinerlei Regelmäßigkeit zu finden sein, und unsere Denkthätigkeit müsste ruhen." [Helmholtz 1879, 41]
} 
aus innerer Anschauung kennen und wissen, durch welche Motive er zu Stande gekommen ist. Von ihm aus beginnt dann, als von einem uns bekannten Anfangsglied und zu einem bekannten Zeitpunkt, die Kette der physischen Ursachen zu wirken, die in den Erfolg des Versuchs ausläuft. Aber eine wesentliche Voraussetzung für die zu gewinnende Überzeugung ist die, daß unser Willensimpuls weder selbst schon durch physische Ursachen, die gleichzeitig auch den physischen Proceß bestimmten, mit beeinflußt worden sei, noch seinerseits die darauf folgenden Wahrnehmungen beeinflußt habe. [Helmholtz 1879, 33; Helmholtz 1896, 594-595] ${ }^{17}$

Der „Willenimpuls“ als einziges nicht determiniertes Glied in der Kette ablaufender Wahrnehmungen ist gleich doppelt in Frage gestellt. Denn der „Willensimpuls" könnte durchaus immer schon unter dem Regime physikalischer Wirkungen und der in Abhängigkeit auftretenden psychischen Effekte stehen. Oder aber er löst nicht nur Handlungen aus, sondern antizipiert schon die Wahrnehmung derselben in bestimmter Weise. Vor diesem Hintergrund werden Träume deutbar, deren kleinste Regungen schon immense physikalisch-symbolische Anstrengungen implizieren und auf deren elegisch symbolische Gesamtschau es überhaupt nicht ankommt:

Der letzte Zweifel kann namentlich bei unserem Thema in Betracht kommen. Der Willensimpuls für eine bestimmte Bewegung ist ein psychischer Act, die darauf wahrgenommene Aenderung der Empfindungen gleichfalls. Kann nun der erste Act den zweiten durch rein psychische Vermittelungen zu Stande bringen? Unmöglich ist es nicht. Wenn wir träumen, geschieht so etwas. Wir glauben träumend eine Bewegung zu vollführen und wir träumen dann weiter, dass dasjenige geschieht, was davon die natürliche Folge sein sollte. Wir träumen, in einen Kahn zu steigen, ihn vom Land abzustossen, auf das Wasser hinauszugleiten, die umringenden Gegenstände sich verschieben zu sehen u.s.w. Hierbei scheint die Erwartung des Träumenden, dass er die Folgen seiner Handlungen eintreten sehen werde, die geträumte Wahrnehmung auf rein psychischem Wege herbeizuführen. Wer weiss zu sagen, wie lang und fein ausgesponnen, wie folgerichtig durchgeführt ein solcher Traum werden könnte. Wenn alles darin im höchsten Grade gesetzmässig der Naturordnung folgend geschähe, so würde kein anderer Unterschied vom Wachen bestehen, als die Möglichkeit des Erwachens, das Abreissen dieser geträumten Reihe von Anschauungen. [Helmholtz 1879, 34; Helmholtz 1896, 594-595]

\footnotetext{
${ }^{17}$ Bemerkenswert ist, daß sich eine solche Sicht des ins Experiment miteingeschlossenen Beobachters wohl nur mit Abstand zu den Experimenten und Versuchsauswertungen selbst einstellt. Denn diese Passage veröffentlichte Helmholtz zunächst anläßlich seiner bekannten Rede und fügte sie dann erst der zweiten Auflage seines Handbuchs für physiologische Optik hinzu. In der ersten Auflage des Handbuchs, die 30 Jahre zuvor erschienen war, ist sie noch nicht enthalten.
} 
So wenig Anstalten Helmholtz auch macht, die psychonome Instanz zu verorten, noch dem subjektiven Idealismus uneingeschränkt das Wort zu reden, so sehr bemüht er Traumvorstellungen als Garanten: Die Möglichkeit einer Sprache ist damit verbürgt, die nicht etwa nur in der Lage wäre, die Naturordnung zu erfassen, sondern ihr gemäß zu wirken.

\section{Bibliographie}

\section{Aristoxenos}

1868 Aristoxenu Harmonikon ta sozomena. Die harmonischen Fragmente des Aristoxenus. Griechisch und Deutsch mit kritischem und exegetischem Commentar und einem Anhang die rhythmischen Fragmente des Aristoxenus enthaltend, hgg. von Paul Marquard, Berlin: Weidmann 1868.

Autrum, Hans Jochem (HG.)

1976 Handbook of Sensory Physiology. Bd. 5: Auditory System. Part 3: Clinical and Special Topics, hgg. von E. de Boer, Berlin, Heidelberg, New York: Springer 1976.

Baxmann, Inge; Franz, Michael; Schäffner, Wolfgang (Hg.) 2000 Das Laokoon-Paradigma. Zeichenregime im 18. Jahrhundert, Berlin: Akademie, 2000.

Buijs-Ballot, Christoph Hendrik Diederik

1845 Akustische Versuche auf der Niederländischen Eisenbahn, nebst gelegentlichen Bemerkungen zur Theorie des Hrn. Prof. Doppler, Annalen der Physik und Chemie, 66, 321-351.

Busch, Oliver

1998 Logos syntheseos. Die Euklidische Sectio Canonis, Aristoxenos, und die Rolle der Mathematik in der antiken Musiktheorie. Im Anhang: Die Teilung des Kanon. Die euklidische Sectio canonis in deutscher Übersetzung, Berlin: Staatliches Institut für Musikforschung — Preußischer Kulturbesitz, 1998.

Cagniard de la Tour, Charles

1819 Sur la Sirène, nouvelle machine d'acoustique destinée à mesurer les vibrations de l'air qui constituent le son, Annales de Chimie et de Physique, 12, 167-171.

CAHAN, DAVID

1993 Hermann von Helmholtz and the Foundations of NineteenthCentury Science, Berkeley, Los Angeles, London: University of California Press, 1993. 
Carnot, Lazare: Berthollet, Claude-Louis

1806 Rapport suivant sur la machine appelée Pyréolophore, ProcèsVerbaux des Séances de l'Académie des sciences tenues depuis la fondation de l'Institut jusqu'au mois d'août 1835, 3, 465-467.

de la Motte-Haber, Helga

1992 Aufbruch in das Klanguniversum, in: [de la Motte Haber 1992, 41-55].

1992 Edgard Varèse: Die Befreiung des Klangs, Hofheim: Wolke 1992. DEIGHTON, LEN

1979 Blitzkrieg. From the rise of Hitler to the fall of Dunkirk, London: Cape, 1979. Zitiert nach der Übersetzung von Hans H. Werner: Blitzkrieg. Von Hitlers Triumphen bis zum Fall von Dünkirchen, Bayreuth: Hestia 1980.

Dostrovsky, Sigalia: Cannon, John T

1987 Entstehung der Musikalischen Akustik (1600-1750), in [Zaminer 1987, 7-79].

FÜCHTBAUER, HEINRICH VON

1947 Georg Simon Ohm. Ein Forscher wächst aus seiner Väter Art, 2. Auflage, Bonn 1947.

Grattan-Guinness, Ivor

1969 Joseph Fourier and the Revolution in Mathematical Physics, Journal of the Institute of Mathematics and its Applications, 5, 230-253.

Heidelberger, Michael

1979 Der Wandel der Elektrizitätslehre zu Ohms Zeit. Eine methodengeschichtliche Untersuchung und logische Rekonstruktion, München: Diss. Phil, 1979.

Helmholtz, Hermann von

1882-1895 Wissenschaftliche Abhandlungen, 3 Bände; Leipzig: Barth, 1882-1895.

1856 Ueber Combinationstöne, Annalen der Physik und Chemie, 99, 497-540. Zitiert nach [Helmholtz 1882-1895, I, 263-302].

1879 Die Thatsachen in der Wahrnehmung. Rede gehalten zur Stiftungsfeier der Friedrich-Wilhelms-Universität zu Berlin am 3. August 1878, Berlin: Hirschwald 1879.

1896 Handbuch der physiologischen Optik, 2. Auflage, Hamburg und Leipzig: Voss 1896.

1913 Die Lehre von den Tonempfindungen als physiologische Grund- 
lage für die Theorie der Musik, 6. Auflage, Braunschweig: Vieweg. KANT, IMMANUEL

1910-1923 Gesammelte Schriften. Erste Abteilung: Werke, hgg. von der Preußischen Akademie der Wissenschaften, 9 Bände, Berlin: Reimer/de Gruyter, 1910-1923.

1781 Kritik der reinen Vernunft. Zitiert nach [Kant, IV, 1-252].

1790 Kritik der Urtheilskraft. Zitiert nach [Kant, V, 165-485].

KITTLER, FRIEDRICH

2000 Eine Kulturgeschichte der Kulturwissenschaft, München: Wilhelm Fink 2000.

Klotz, Sebastian

2000 Tonfolgen und die Syntax der Berauschung. Musikalische Zeichenpraktiken 1738-1788, in [Baxmann, Franz, Schäffner 2000, 306338].

Kuhn, Thomas

1961 Sadi Carnot and the Cagnard Engine, Isis, 52, 567- 574.

Leibniz, Gottfried Wilhelm

1765 Nouveaux essais sur l'entendement par l'auteur du systeme de l'harmonie preestablie, in: Die philosophischen Schriften, hgg. von Carl Immanuel Gerhardt, Bd. 5, Reprint Hildesheim: Georg Olms 1996.

LENOIR, TIMOTHY

1993 The Eye as Mathematician: Clinical Practice, Instrumentation, and Helmholtz's Construction of an Empiricist Theory of Vision, in: [Cahan 1993, 109-153].

1994 Helmholtz and the Materialities of Communication, Osiris, 9, 185-207.

MaCh, ERnst

1860 Über die Änderung des Tones und der Farbe durch Bewegung, Sitzungsberichte der Mathematisch-Naturwissenschaftlichen Classe der Kaiserlichen Akademie der Wissenschaften (Wien), 41, 543560 .

1861 Ueber die Controverse zwischen Doppler und Petzval, bezüglich der Aenderung des Tones und der Farbe durch Bewegung, Zeitschrift für Mathematik und Physik, 6, 120-126.

1862 Ueber die Aenderung des Tones und der Farbe durch Bewegung, Annalen der Physik und Chemie, 116, 333-338.

MÜLLER, JOHANNES

1833-38 Handbuch der Physiologie des Menschen, 2 Bände; Coblenz: 
Hölscher 1833-38.

Neumaier, Wilfried

1986 Was ist ein Tonsystem? Eine historisch-systematische Theorie der abendländischen Tonsysteme, gegründet auf die antiken Theoretiker Aristoxenos, Eukleides und Ptolemaios, dargestellt mit Mitteln der modernen Algebra, Frankfurt am Main, Bern, New York: Peter Lang, 1986.

Oesch, Hans

1954 Guido von Arezzo. Biographisches und Theoretisches unter besonderer Berücksichtigung der sogenannten odonischen Traktate, Bern: Haupt 1954.

Ohm, Georg Simon

1843 Ueber die Definition des Tones, nebst daran geknüpfter Theorie der Sirene und ähnlicher tonbildender Vorrichtungen, Annalen der Physik und Chemie, 59, 513-565.

1844 Noch ein paar Worte über die Definition des Tones, Annalen der Physik und Chemie, 62, 1-18.

PAYEN, JACQUES

1971 Cagniard de la Tour, in: Dictionary of Scientific Biography, hgg. von Charles Coulston Gillespie, Bd. 3, New York: Charles Scribner's Sons, 1971.

RhEINBERGER, HANS-JÖRG

2001 Experimentalsysteme und epistemische Dinge. Eine Geschichte der Proteinsynthese im Reagenzglas, Göttingen: Wallstein 2001.

SiEgERT, BERnHARD

2000 Schüsse, Schocks und Schreie. Zur Undarstellbarkeit der Diskontinuität bei Euler, d'Alembert und Lessing, in [Baxmann, Franz, Schäffner 2000, 291-305].

Turner, STEVEN

1977 The Ohm-Seebeck Dispute, Hermann von Helmholtz, and the Origins of Physiological Acoustics, British Journal for the History of Science, 10, 1-24.

VIRILIO, PAUL

1984 Guerre et cinéma, Paris: Ed. De l'Etoile, 1984. Zitiert nach der Übersetzung von Frieda Graefe: Krieg und Kino, Logistik der Wahrnehmung, München, Wien: Hanser 1986.

Vogel, Stephan

1993 Sensation of Tone, Perception of Sound, and Empiricism: Helmholtz's Physiological Acoustics, in: [Cahan 1993, 259-287]. 
Wundt, WiLhelm

1901 Gustav Theodor Fechner - Rede zur Feier seines hundertjährigen Geburtstages, Leipzig: Engelmann, 1901.

ZAminer, Frieder (HG.)

1987 Geschichte der Musiktheorie. Bd. 6: Hören, Messen und Rechnen in der frühen Neuzeit, Darmstadt: Wissenschaftliche Buchgesellschaft, 1987.

ZHMUd, LEONID

1994 Nauka, filosofija i religija v rannem pifagoreizme, Sankt Petersburg: VGK, 1994. Zitiert nach der Übersetzung des Autors: Wissenschaft, Philosophie und Religion im frühen Pythagoreismus, Berlin: Akademie, 1997.

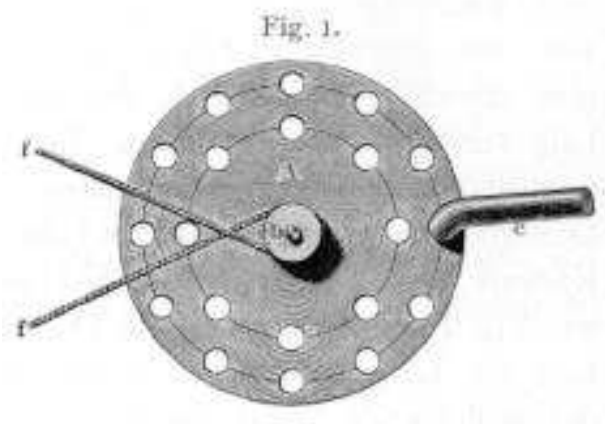

Abbildung 1: Das Prinzip der Sirene blieb während der ganzen Zeit ihrer experimentellen Anwendung im Grunde gleich. Nur verzichtete man sehr bald auf Cagniards Realisierung des Antriebs der Scheibe durch den Luftstrom und sah statt dessen einen unabhängigen Antrieb vor, um mit der Erhöhung des Luftdrucks eine Erhöhung der Lautstärke zu erreichen ohne gleichzeitig die Frequenz der angeblasenen Löcher und damit einen Anstieg der Tonhöhe zu verursachen. Helmholtz gibt in seiner Lehre von den Tonempfindungen eine präzise Beschreibung der Sirene: "A ist eine dünne Scheibe aus Pappe oder Blech, welche um ihre mittlere Achse b mittels der um ein größeres Rad laufenden Schnur ff schnell gedreht werden kann. Längs des Randes der Scheibe ist eine Reihe von Löchern in gleichen Abständen von einander angebracht, in der Zeichnung 12; eine oder mehrere andere Reihen gleichabstehender Löcher befindet sich auf anderen konzentrischen Kreislinien [hier acht Löcher]; c ist ein Röhrchen, 
welches gegen eines der Löcher gerichtet wird. Läßt man nun die Scheibe geschwind umlaufen, und bläst durch das Röhrchen c, so tritt die Luft frei aus, so oft eines der Löcher der Scheibe an der Mündung des Röhrchens vorbeigeht, während der Austritt der Luft gehindert ist, so oft ein undurchbohrter Teil der Scheibe vor der Mündung des Röhrchens steht." [Helmholtz 1913, 21]
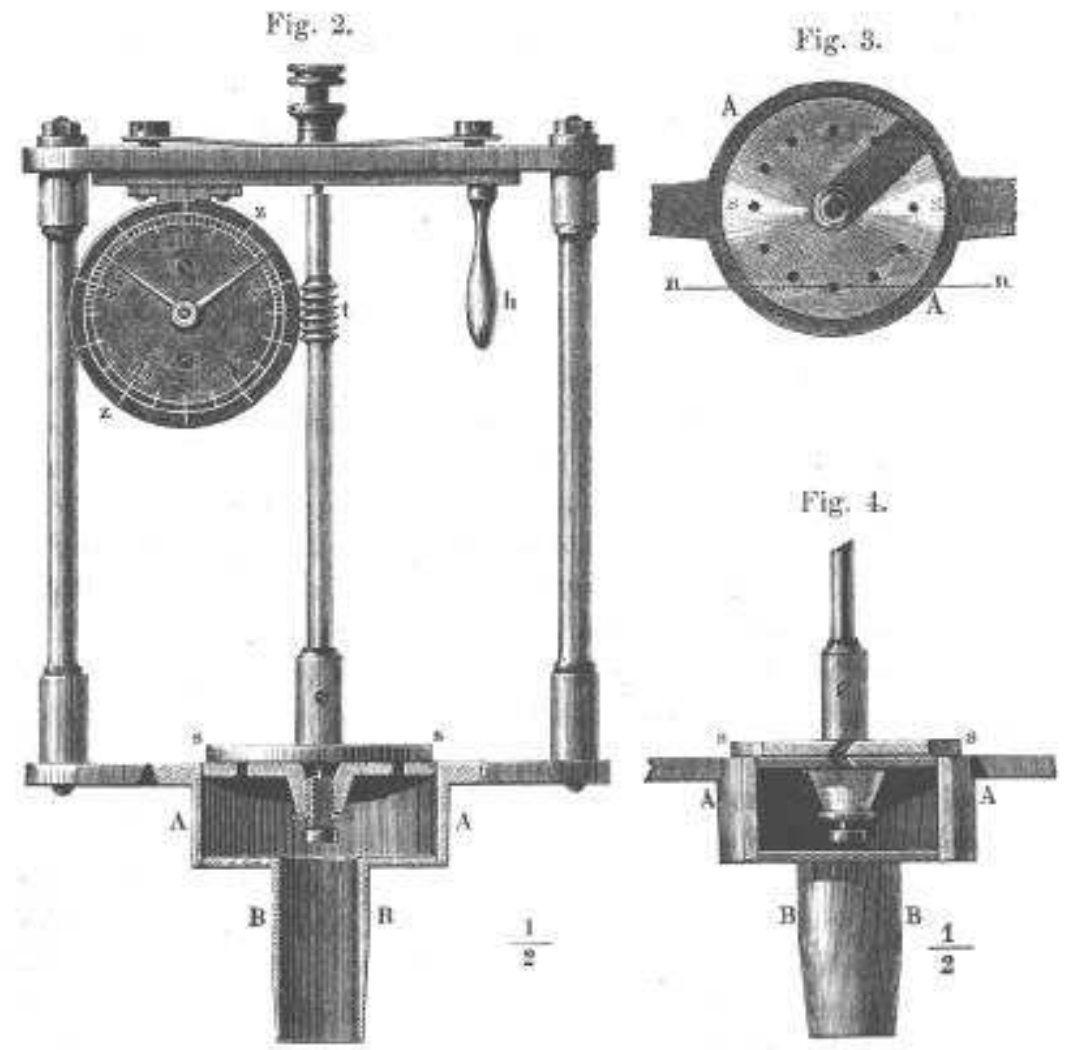

Abbildung 2: Zu sehen ist die Darstellung von Cagniards Sirene, wie sie Helmholtz in seiner "Lehre von den Tonempfindungen“ gibt [Helmholtz 1913, 23]. Die Komprimierung der Luft in einem Windkasten A und die Anzahl der Löcher in demselben, die mit der Scheibe übereinstimmt, bringen einen sehr lauten Ton zustande. Der Schnitt durch das Instrument in Fig.4 zeigt wie durch schräg aufeinander gerichtete Luft- 
kanäle die Scheibe durch den Luftstrom angetrieben werden kann. In Fig.2 ist ein einstellbares Zählwerk z zu erkennen, das die Umdrehungen der Scheibe mißt.

\begin{tabular}{|c|c|c|c|}
\hline 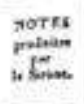 & 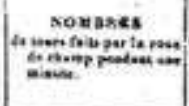 & 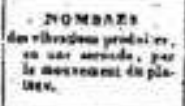 & 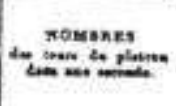 \\
\hline (1) La & 19 & 427 & $4 \div$ \\
\hline Sir & $21 \div$ & 477 & $4 \frac{21}{x+a}$ \\
\hline$U_{t}$ & $22 \frac{3}{2}$ & 511 & $5 \frac{11}{12}$ \\
\hline $11 \hat{e}$ & 25 & 567 & $5 \div$ \\
\hline Mi & as & 650 & $6 \frac{26}{17}$ \\
\hline $\mathrm{Fa}$ & 50 & 6,5 & $6 \frac{73}{104}$ \\
\hline Sol & 34 & 765 & $7 \frac{58}{104}$ \\
\hline 1.2 & 38 & 955 & $8 \frac{\mathrm{cs}}{\mathrm{wat}}$ \\
\hline $\mathbf{S}_{i}$ & $42 \div$ & 955 & $9 \frac{32}{10}$ \\
\hline $\mathbf{U}_{l}$ & $45 \div$ & 1003 & 10 尚 \\
\hline Ad & 50 & 1125 & $14 \frac{45}{400}$ \\
\hline
\end{tabular}

Abbildung 3: Tabelle Cagniards de la Tours in seinem Bericht zur Sirene von 1819. Gegenübergestellt sind die verschiedenen Noten und ihre Schwingungsanzahl pro Sekunde, hervorgerufen durch eine bestimmte Anzahl an Umdrehungen der Scheibe der Sirene [Cagniard de la Tour $1819,171]$. 